\title{
Cognitive Media Access Control
}

\author{
Po-Yao Huang \\ National Taiwan University \\ Taiwan
}

\section{Introduction}

With the increasing demand of wireless communication and the fact of under utilization of wireless spectrum, a lot of researches have developed technologies to enhance the efficiency of spectrum utilization. The spectrum allocation chart by the Federal Communications Commission (FCC) (NTIA, 2003) indicates high degree legislation of frequency band, in other words, our sky is quite crowded. However, another study by FCC (Report \& Order, 2002) also points out the fact that the conventional, fixed spectrum allocation policy is inadequate in addressing nowadays rapidly growing wireless communication. Vast temporal and geographic variations in the usage of allocated spectrum utilization ranging are from $5 \%$ to $75 \%$ below $3 \mathrm{GHz}$ while the spectrum utilization is even worse in the range above $3 \mathrm{GHz}$. Generally speaking, many licensed spectrum blocks are idle at most of the time. These unused spectrum blocks are known as spectrum holes, which is defined in (Haykin, 2005) : A spectrum hole is a channel (i.e. frequency band) assigned to a primary user, but, at a particular time and specific geographic location, the channel is not being utilized by that primary user. To resolve the spectrum inefficiency in the more demanding reality, dynamic spectrum access (DSA) techniques are proposed to resolve the challenge of insufficient spectrum utilization as investigated in (Akyildiz et al., 2006) and (Jha et al., 2011).

\subsection{Cognitive radio networks}

The key to enable DSA is cognitive radio (CR) (Mitola \& Maguire, 1999) technology, which is conventionally defined as a physical/link-level technology aims at realizing DSA. In the standardization as IEEE 802.22 (IEEE, 2009), CR has been considered as a promising technology to enhance spectrum/channel efficiency while primary systems (PSs) are with relatively low spectrum utilization. CR provides the capability to share the channel with PSs' users in a opportunistic way. Moreover, CR can further provide networking "macro-scale diversity" above link layer to bridge the integrated re-configurable system networking vision as described in (Chen et al., 2008). Such a scenario for future wireless networks is recognized as cognitive radio networks (CRNs) which are envisioned to provide high bandwidth and good quality of service (QoS) to mobile users via heterogeneous wireless network architectures by reconfigurable CR transceiver for DSA. CRN can be deployed in network centric, fully distributed, Ad-hoc, and mesh architectures and serve both licensed and unlicensed applications. The basic component of CRN are model station (MS), base station/access point (BS/AP), and backbone/core networks. These components construct three kinds of network architectures in CRN : Infrastructure, ad-hoc, and mesh networks. 
There are generally two kinds of wireless communication systems in CRN : primary system (PS) and cognitive radio (CR) system. They are classified by their properties on a specific channel. In general, a PS is licensed. PS is an existing system operating on the channel and has a higher priority to utilize its channel than other CRs. On the contrary, CRs, which are capable to switch over multiple channels, facing the new challenges to exploit the spectrum holes over multichannel as well as avoid interfering PSs simultaneously. These new challenges define a new media access control (MAC) problem under CR paradigm. With different PSs, the inherent characteristic in the MAC of CRN is a multichannel scenario with high priority PSs' users and competing CRs. In next section, we will first discuss the conventional multichannel MAC problem followed by the new challenge of MAC under CR paradigm.

\subsection{Conventional media access control}

Before introducing the $\mathrm{MAC}$ for $\mathrm{CR}$, we start from the conventional MAC problem (Bertsekas \& Gallager, 1992). As it contents, stations share a common media(i.e.,channel)and try to communicate on the shared channel. (Note that the term "station" is used in this subsection of discussion in comparison of "CR" in following sections). Without losing generality, the original MAC problem with $N$ devices/stations in local area network (LAN) can be shown as Fig. 1-(a).

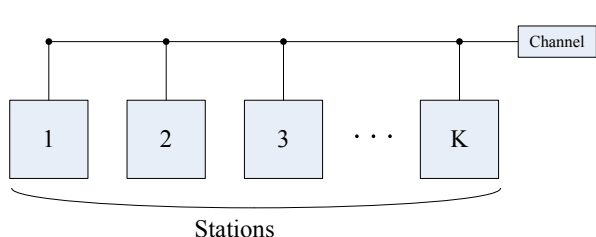

(a) Conventional MAC

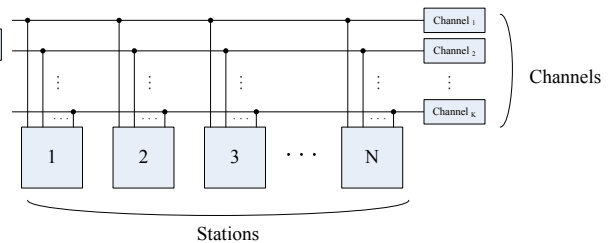

(b) Multichannel MAC

Fig. 1. Subfigure with four images

All stations share a common media and try to utilize the media for transmission. If more than two stations try to access the shared media simultaneously, then collision happens. In other words, there is contention between stations for the limited resource. Many protocols have been developed to resolve this problem such as CSMA/CD in local area network (LAN) and CSMA/CA in wireless LAN (WLAN). As discussed in (Marsan \& Roffinella, 1983), multiple orthogonal channels provide a new degree of freedom to further alleviate the contention. Multichannel MAC, depicted as Fig. 1-(b), is considered to utilize multiple orthogonal channels for throughput enhancement by allowing parallel transmissions over channels. The conventional design of multichannel MAC under wired LAN is simple, stations equipped with multiple volt-meters may tuned to access those multichannel(separated wires or orthogonal frequency bands). Collision still happens if there is a co-channel transmission simultaneously.

A more critical and challenging scenario lies in the multiple wireless networks, such as dynamic spectrum access over multiple wireless communication systems shown in Fig. 2 . The main difference between wired and wireless networks is that now stations cannot access multiple systems simultaneously under hardware design restrictions. At a specific time period, station can only select and switch to one specific channel for communication. Therefore, the wireless multichannel MAC problem is further been divided into two parts. 
The first part is "Collision Avoidance/Resolution." Collision avoidance/resolution (Sun et al., 1997) is inherited from conventional single channel MAC problem. Contention must be avoided/resolved when more than two stations try to access the channel. Stations must avoid simultaneous transmission and resolve further possible failure after collision happens. On the other hand, with the limited hardware capability for channel access, a stations may listen/transmit over one selected channel. Therefore, a scheme/protocol framework must be properly designed for multiple stations to select the proper channel for data transmission. To achieve so, the brand new problem lies in wireless multichannel MAC is the "Channel Selection" part. Channel selection, which considers distributed selection of communication channel, is the new challenging issue which recently attracts most efforts as in (Mo et al., 2008).

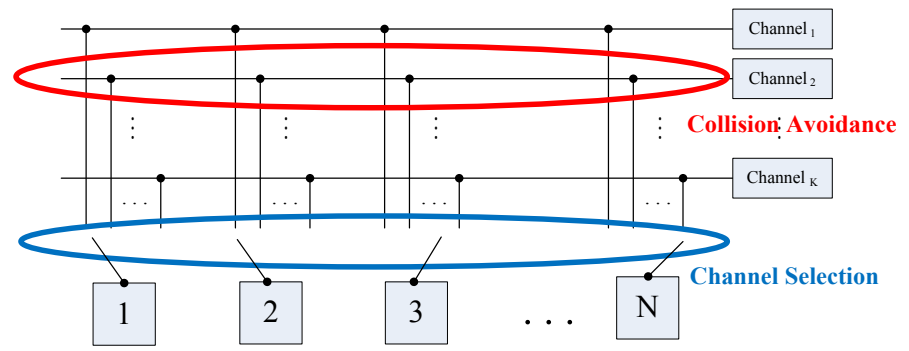

Fig. 2. Two fundamentals of Multichannel MAC : 1) Collision Avoidance/Resolution and 2)Channel Selection.

\subsection{Cognitive media access control for CR}

The generalized multichannel MAC under CR paradigm can be illustrated in Fig. 3. In CRN, CRs are categorized as secondary users who sense for the spectrum/channel opportunity and access the channel with a lower priority than PSs' users. Since CR and PS belong to different systems, the feasible multichannel MAC protocol for $C R$ is required to resolve not only intra-system (CR-CR, within CRN) but also inter-system (CR-PSs) media contention. Under the design limitation that each CR cannot access all of the available channels simultaneously (i.e., CR equipped with only one reconfigurable transceiver/radio), the main task of CR MAC is to distributively choose the channel to use without a reserved common control channel.

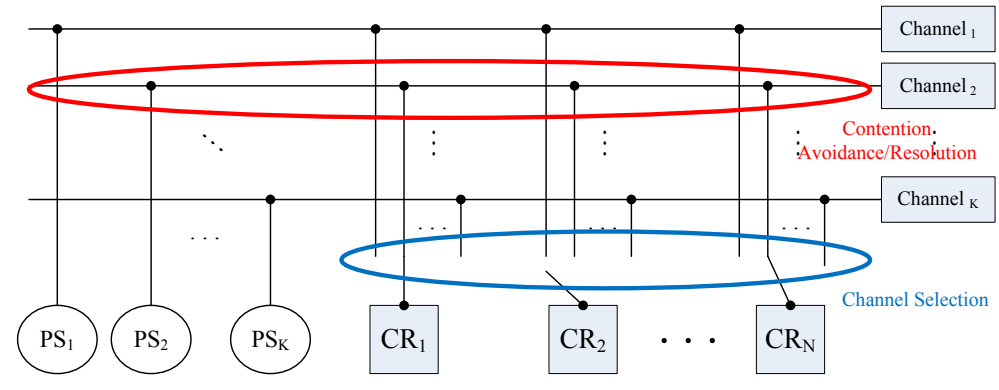

Fig. 3. CR multichannel MAC 
Based on the discussion above, CR multichannel MAC differs from conventional wireless multichannel MAC problem. CRs, as secondary users, must agilely avoid interference with prior communication users. Moreover, for support of multiple access among CRs, a contention avoidance/resolution mechanism must be properly designed. To further enhance the spectrum efficiency and throughput of CRN, a feasible channel access strategy should also be properly designed. Therefore, the aims of CR multichannel MAC are :

1. Efficiently utilizing multichannel by initiating parallel transmissions in a distributive manner.

2. Meeting the requirement that transmissions of CRs are interference-free for PSs.

3. Alleviating contention over CRs and PSs, maximizing the CRN throughput.

Moreover, $\mathrm{CR}$, which is envisioned to be equipped with sensing and cognitive capability, brings new possibility for further performance enhancement under its challenging multiple co-existing PSs MAC scenario. CR, with ability to retrieve environmental information and learn to adapt itself, is possible to achieve the optimal performance. For example, the stabilization under $\mathrm{CR}$ multichannel MAC is a more complicated problem then conventional single channel solution (Limb, 1987). Take stabilization as an example, 1) Intra-system (CR-CR, within CRN) information : the load distribution over multiple channels and 2) Inter-system (CR-PSs) information behaviour of PSs can be retrieved by cognitive functionality of $\mathrm{CR}$ for further throughput optimization. The related cognitive functionality will be introduced, analysed and discussed in the following sections.

In this chapter, we will develop a general protocol scheme for CR multichannel MAC. A CSMA-based cognitive MAC is proposed with a general Markov Chain analysis and the steady state approximation for practical design insight. The cognitive functionality of $C R$ and optimization is also proposed for MAC protocol optimization. The simulation results validate the analysis, and are compared with the results with current research works.

This chapter is organized as follows Section II summaries the related work. Section III describes the system model under consideration. The proposed general CR multichannel MAC scheme is in Section IV with a CSMA-based cognitive MAC design. In Section V, we model, analyse and provide a steady-state protocol performance approximation. Cognitive functionality and performance enhancement and optimization is introduced in Section VI. Section VII is the simulation results and discussion. Conclusion and future works are presented in section VIII.

\section{Multichannel MAC: theory and related works}

Representative multichannel MAC protocols are summarized in Table 1. These protocols can further be categorized as narrow-band approaches and wide-band approaches. For narrow-band transmitter and receiver, the frequency band to be used for transmission is to be predetermined, or dynamically chosen. While in wide-band system, the transmitter can simultaneously transmit over multiple frequency bands that are detected to be unoccupied while the receiver can retrieve information over multichannel simultaneously. Note that in wide-band systems, each station may need more than two radios to achieve the parallel transmissions over multichannel while in narrow-band system, a station is equipped with only one radio. (Two radios in dedicated control channel multichannel MAC case.) These multichannel protocols can further be categorized as follows: 


\begin{tabular}{|c|c|c|}
\hline \multicolumn{3}{|c|}{ The Related Works } \\
\hline Category & Protocol Type & Reference (Year \\
\hline \multirow{4}{*}{ Narrow-Band } & Dedicated Control Channel & $\begin{array}{c}\text { (Wu et al., 2000) } \\
\text { (Zhang \& Su, 2011) } \\
\text { (WC Hung, 2002) } \\
\text { (Cordeiro \& Challapali, 2007) } \\
\text { (Liu \& Ding, 2007) } \\
\text { (Timmers et al., 2007) }\end{array}$ \\
\hline & Common Hopping & $\begin{array}{c}\text { (Tang \& Garcia-Luna-Aceves, 1998) } \\
\text { (Tzamaloukas \& Garcia-Luna-Aceves, 2000) }\end{array}$ \\
\hline & Split Phase & $\begin{array}{c}\text { (Chen et al., 2003) } \\
\text { (So \& Vaidya, 2004) } \\
\end{array}$ \\
\hline & Multiple Rendezvous & $\begin{array}{l}\text { (Bahl et al., 2004) } \\
\text { (Mo et al., 2008) }\end{array}$ \\
\hline \multirow[b]{2}{*}{ Wide-Band } & CDMA/Frequency Coding & $\begin{array}{c}\text { (Al-Meshhadany \& Ajib, 2007) } \\
\text { (Zhang et al., 2003) }\end{array}$ \\
\hline & Multi-Radios & $\begin{array}{c}\text { (Nasipuri et al., 1999) } \\
\text { (Jain et al., 2001) }\end{array}$ \\
\hline
\end{tabular}

Table 1. Related Works

\subsection{Protocols with dedicated control channels}

As shown in Fig. 4-(a), one (or more) control channels are utilized to exchange control signal (such as request to send (RTS) and clear to send (CTS) in WLAN) and distributed sensing (Liu \& Ding, 2007) or cooperative sensing (Zhang \& Su, 2011) information of CRs. CR using this type of protocol must equip with at least two radios, one (or more) radio is reserved to listen to the control channel. It should be noted that the reservation of a common control channel violates $\mathrm{CR}$ design philosophy which utilize the spectrum holes in a opportunistic sense. The performance bottleneck of this kind of protocol is the overhead over the reserved common control channel.

\subsection{Protocols with common hopping}

In protocols with common hopping, CRs hop across multichannel following the same hopping pattern (Tang \& Garcia-Luna-Aceves, 1998),(Tzamaloukas \& Garcia-Luna-Aceves, 2000). As shown in Fig. 4-(b), common hopping is comparable to "switch" control channels over multichannel. The bottleneck of such protocol type is still the overhead of the hopping control channel. This type of protocol requires only one radio.

\subsection{Split phase protocols}

Depicted in Fig. 4-(c), time is divided into control and data phases (Chen et al., 2003), (So \& Vaidya, 2004). CR exchange control information on a dedicated control channel during the control phase, then accessing the channel during the data phase. The idea is analogous to "reserve" some time duration as control channel. The bottleneck is still the overhead of the control channel. One radio is required using this type of protocol. 


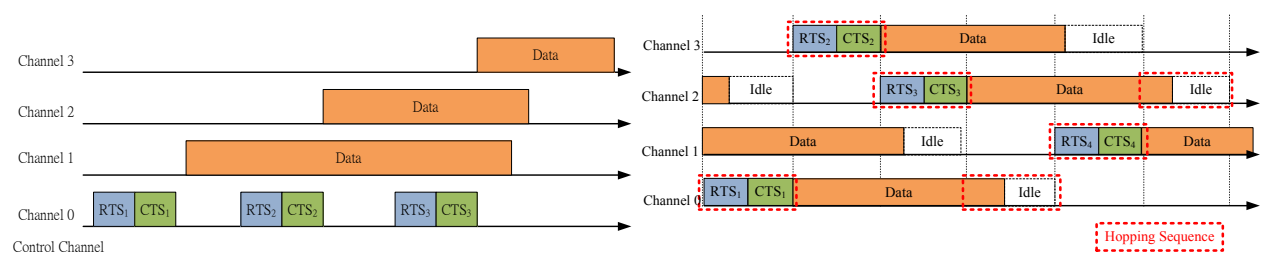

(a) Dedicated Control Channel MAC

(b) Common Hopping MAC

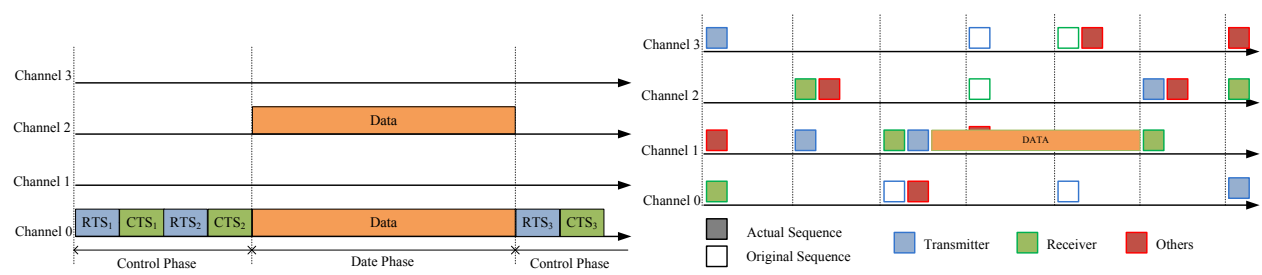

(c) Split Phase MAC

(d) Multiple Rendezvous MAC

Fig. 4. Related Multichannel MAC Protocols

\subsection{Multiple rendezvous protocols}

In (Bahl et al., 2004) and (Mo et al., 2008), CRs opportunistically select a channel, exchange control information then transmit data. Shown in Fig. 4-(d), the main idea is comparable to "separate" control channel over multichannel. Multiple transmissions can be ignited over channels with only one radio. Parallelism is the design philosophy of this kind of protocol. However, the cons of these results are the lack of joint consideration of channel selection and collision avoidance/resolution of inter-system (PS-CR) and intra-system (CR-CR). The performance is degraded under PSs presentation and contention over CRs.

From the discussion above, one should note that for the feasible CR realization, the preserved common control channel should be avoided since it violates the design philosophy of CR. Furthermore, CR with only one transceiver is more practical design. In the following section, we provide a general model for analysis of multichannel MACs and propose the cognitive multichannel MAC which optimizes CRN throughput performance by enabling multiple rendezvous and fits requirements of multichannel MAC under CR paradigm.

\section{System model}

\subsection{Network model}

The considered generalized CR multichannel MAC is a fully-distributed ad-hoc network as in (Mo et al., 2008). The infrastructure scenario under CRN paradigm is exampled and illustrated in Fig. 5. There are multiple co-existing PSs, while CRs tries to utilize multichannel to communicate with other CRs or base station of CRN. Physically, CRs are capable to reconfigure its physical layer to access every channel selected from the pool of multichannel where different PSs pre-occupy. Categorized as secondary users, CRs seek channel opportunities over multichannel and access the channel to relay its data to the backbone network or other CRs without interfering PSs' users. 


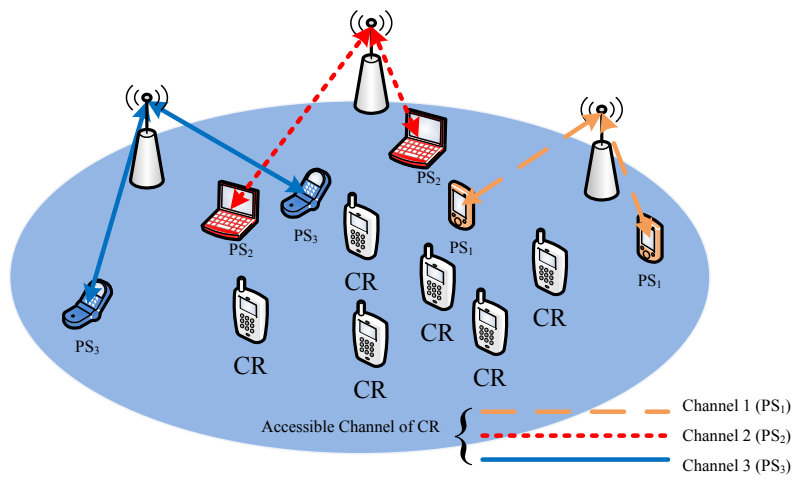

Fig. 5. CR System Model

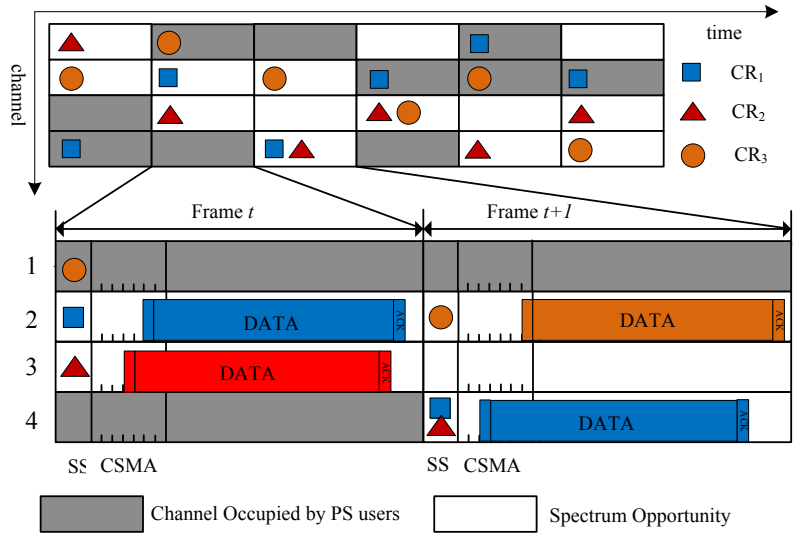

Fig. 6. Cognitive CSMA-based McMAC protocol for CRN

\subsection{Multichannel MAC under CR paradigm}

The general CR multichannel MAC is depicted in Fig. 6. Time is divided into small synchronized ( $t$ as the frame index) slots over multichannel. The duration of a slot/frame is denoted as $t_{l}$. The CR multichannel MAC is characterized by: $\{K, N, C, \mathbf{q}\} . K$ is defined as the number of multiple channels, we use the set $\{k\}$ as the pool for channel selection. The switching of channel take place only at slot boundary. Each channel $k$ is supposed to be independently pre-occupied by some licensed PS or by CR. CR access the channel only if there is no PS appearance and vice versa. The appearance of PS on channel can be represented as a on-off two-state Bernoulli process with parameter $q_{k}$. We define $\mathbf{q} \triangleq\left\{q_{k} \mid k=1,2 \ldots K\right\}$ as the set of probabilities of PSs' appearance over channel $1 \ldots k$.

The number of CRs is denoted as $N$. The value of $N$ can be estimated by neighbour discovery, network initialization algorithms as in traditional study of Ad-Hoc network as in (RoyChoudhury et al., 2000) and (Jakllari et al., 2005). Through this chapter we assume this value is correctly estimated by CRs in a distributed manner with no transient behaviour. The average capacity over channels which indicates the maximum throughput per slot is denoted as $C$. 
The performance indicator for CR multichannel MAC paradigm are 1) the aggregated throughput $R$, defined as the aggregated physical layer throughput over all channels, and the 2) average channel utilization $U$, defined as the average number of channels being successfully utilized for data transmission, normalized by the number of channel. Note that $U$ is equivalent to traditional MAC "throughput" defined as the number of successful transmission per channel per slot. The notations of generalized CR multichannel MAC are summarized in Table 2.

\section{General CR multichannel MAC protocol}

There are two components in multichannel MAC: 1) Channel Selection and 2) Contention Avoidance/Resolution. The general CR multichannel MAC and its frame structure is illustrated in Fig. 6. At the beginning of a frame, CR selects a channel with the channel selection strategy, denoted as $\Gamma$. In the frame operation, the contention avoidance/resolution is composed of two parts: 1) spectrum sensing for inter system (CR-PS) contention avoidance/resolution; and 2) intra system (CR-CR) contention avoidance/resolution mechanism. The mathematical form of these process can be represented by a contention avoidance/resolution function $g_{k}(n)$. With feasible channel selection and contention avoidance/resolution mechanisms, CR proceed reliable transmission.

\subsection{Contention avoidance/resolution algorithm $g_{k}(n)$}

The general form of contention avoidance/resolution can be represented by a resolution function $g_{k}(n)$ defined as:

$$
g_{k}(n)=\operatorname{Prob}\left(S_{k}=1 \mid n \text { CRs attempt to transmit over channel } k\right)
$$

$S_{k}$ is the indicator that channel $k$ is successfully utilized by CR. The resolution function $g_{k}(n)$ can further be divided into two parts: 1) the availability $\phi_{k}$ of channel $k$, that is, the channel must be free form PSs' users. 2) The contention resolution mechanism among CRs. Define $1_{k}(t)$ as the indicator denoting the availability of $k$ th channel at slot $t$ :

$$
1_{k}(t)= \begin{cases}1, & \text { channel } k \text { is available at slot } t \\ 0, & \text { otherwise. }\end{cases}
$$

Spectrum sensing is performed to acquire $\left(1_{k}(t)\right)$ of CR's currently locating channel . As a detection problem, let $P_{f}$ and $P_{d}$ are probability of false alarm and probability of detection, respectively. Therefore, $\phi_{k}=\left(1-q_{k}\right)\left(1-P_{f}^{k}\right)+q_{k}\left(1-P_{d}^{k}\right)$, where $\phi_{k}$ is defined as the channel availability. For simplicity of MAC analysis, we assume perfect spectrum sensing, that is, $P_{f}=0$ and $P_{d}=1$, Therefore, $\phi_{k}=1-q_{k}$. Link the indicator function and the channel availability $\phi_{k}$, we have:

$$
\left\{\begin{array}{l}
\operatorname{Prob}\left(1_{k}=1\right)=\phi_{k}=1-q_{k} \\
\operatorname{Prob}\left(1_{k}=0\right)=1-\phi_{k}=q_{k}
\end{array}\right.
$$

After spectrum sensing, contentions among CR are further alleviated with contention avoidance/resolution mechanisms. Many conventional works have been proposed to resolve this problem such as 1-persistent CSMA, p-persistent CSMA, non-persistent CSMA, etc. The contention avoidance/resolution in this part can be slotted or non-slotted. In the later section, a non-persistent CSMA mechanism is proposed and analysed. 


\begin{tabular}{|c|l|c|l|}
\hline Note & Description & Note & Description \\
\hline$K$ & Number of Channel & $N$ & Number of CR \\
\hline $\mathbf{C}$ & Set of Channel Capacity & $\mathbf{q}$ & Set of Prob. of PS Appearance \\
\hline$C$ & Average Channel Capacity & $k$ & index of channel \\
\hline$t$ & Frame Index & $t_{l}$ & Frame Length \\
\hline$q_{k}$ & Prob. of PS on Channel $k$ & $p_{k}$ & Prob. of Selecting Channel $k$ \\
\hline$\Gamma$ & Channel Selection Strategy & $R$ & Aggregated CR Throughput \\
\hline$U$ & Utilization & $X_{t}$ & $\#$ of CR Transmission at slot $\mathrm{t}$ \\
\hline $\mathrm{S}$ & State Space of X & $i$ & $\#$ of current CR Transmission \\
\hline$j$ & $\#$ of CR Starting/Termination & $P_{i j}$ & Transition Prob. \\
\hline $\mathbf{P}$ & Transition Prob. Matrix & $\pi_{i}$ & Limiting Prob. of X \\
\hline$\Pi$ & Limiting Prob. Vector & $S_{i}^{j}$ & Prob. of Starting $j$ Transmission \\
\hline$S_{i}^{j}$ & Prob. of Terminating $j$ Transmission & $t_{l}$ & Slot Length \\
\hline$s_{k}$ & Duration of Spectrum Sensing & $t_{k}$ & Duration of Control Message \\
\hline$\eta_{k}$ & Protocol Efficiency over Channel $k$ & $\phi_{k}$ & Availability of Channel $k$ \\
\hline
\end{tabular}

Table 2. Notation of Generalized Multichannel MAC for CRs

\begin{tabular}{|c|c|c|}
\hline Category & Protocol Name & Selection Algorithm \\
\hline Deterministic & Best Channel & $p_{k}=1$ where $k=\operatorname{argmax} \phi_{k}$ \\
\hline Random & Uniform Selection & $p_{k}=\frac{1}{R}$ \\
\hline \multirow{2}{*}{ General } & Proportional Selection & $p_{k}=\frac{\phi_{k}}{\sum_{i} \phi_{i}}$ \\
\cline { 2 - 3 } & Optimal Selection & $p_{k}=p *($ Section 6) \\
\hline
\end{tabular}

Table 3. Channel Selection Algorithms

\subsection{Channel selection algorithms $\Gamma$}

Without a reserved control channel for exchanging control information, CR distributedly selects multichannel with a channel selection algorithm $\Gamma$. Its effect can be analogous to separate CR traffic over multichannel. The resulting contention over a specific channel is thus alleviated. The general channel selection algorithm is defined as $\Gamma=\left\{p_{1}, p_{2}, \ldots, p_{K}\right\}=\left\{p_{k}\right\}$ and $\sum_{k} p_{k}=1$, where $p_{k}$ is the probability of selecting channel $k$. The category and details of channel selection algorithms are summarized in Table 3.

\subsection{Protocol description}

As shown in Fig. 6. With swift channel opportunity, transmission are initiated and terminated within one frame in avoidance of further interference with PSs. Moreover, With the cognitive functionality powered by sensing and adaptation of CRs, the cognition of $q_{k}$ and $N$ can be further utilized in MAC protocol optimization. The proposed cognitive multichannel MAC protocol are stated as below:

1. (Channel Selection) If CR attempts to transmit, at the beginning of a frame, the channel $k$ is selected with probability $p_{k}$.

2. (Spectrum Sensing Phase) Perform spectrum sensing to detect the appearance of specific PS on its currently locating channel $(k)$ and update the probabilities of PS appearance q.

(a) If PS appears, update $q_{k}$ and skip step (3) and (4).

(b) Else, update $q_{k}$ and proceeds. 
3. (CSMA-contention Phase) CR transmitter pick a back-off value between $\left[0, N_{c w}\right]$, where $N_{c w}$ is the contention window size. CR transmitter decrements its back-off value by one during each idle slot. In this phase, CR keeps listening to the channel.

(a) If the channel becomes busy before the back-off value reach 0 , gives up transmission.

(b) Else, sets access indicator $(S=1)$, access the channel.

4. (Data transmission Phase) Transmit data. Transmission terminated before end of frame.

(a) $\operatorname{If}(S=1)$, Transmit data; terminates after ACK.

(b) Else if the data is correctly received, transmits ACK.

(c) Otherwise, remains silent until the end of frame.

\section{Protocol performance evaluation}

The performance of the proposed general protocol is evaluated with Markov chain and a steady-state approximation to build intuition of parameter dependence for further optimization process in practical realization. Supposed that that CR are synchronized with PSs and spectrum sensing is perfectly performed (i.e., if PS appears at frame $t$, all the CRs who tuned the channel detect its appearance and avoid channel access.) The packet arrival of CR is assumed to follows a Poisson Process with parameter $\lambda$. The retransmission policy follows a geometric back-off with parameter $q_{r}$. If CR is backlogged, with probability $q_{r}$ it attempts channel access to retransmit the backlogged packet.

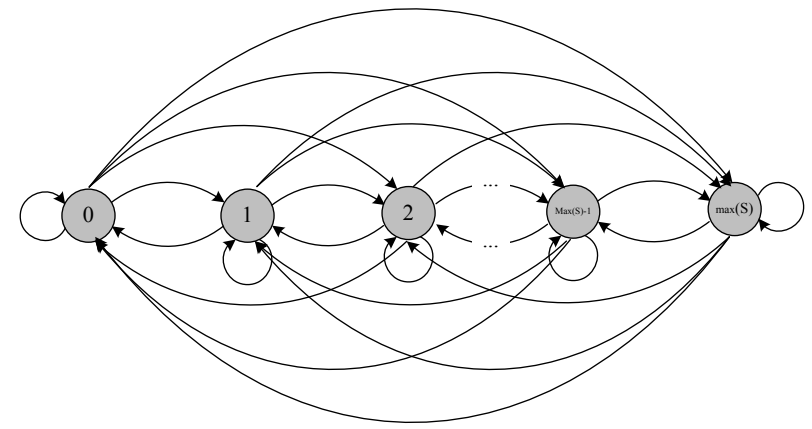

Fig. 7. The Markov Chain Model for multichannel MAC

\subsection{The Markov chain model}

Let $X_{t}$ denote the number of backlogged CRs at the beginning of a given frame $t . X_{t}$ changes from frame to frame and $\left\{X_{t}\right\}$ forms a discrete time Markov chain as in Fig. 7. The state space of $\left\{X_{t}\right\}$ is defined as: $\mathbb{S}=\{0,1, \ldots, N\}$ Let $Q(x, i)$ be the probability that $i$ un-backlogged CRs attempt to transmit packets in a given frame, and that $Q_{r}(y, i)$ be the probability that $i$ backlogged node transmit. We have:

$$
Q_{a}(x, i)=\left(\begin{array}{c}
N-i \\
x
\end{array}\right)\left(1-q_{a}\right)^{N-i-x} q_{a}^{x}
$$

and

$$
Q_{r}(y, i)=\left(\begin{array}{l}
i \\
y
\end{array}\right)\left(1-q_{r}\right)^{i-y} q_{r}^{y}
$$


Where $q_{a}=1-e^{-\lambda t_{l}}$ is the probability of packet arrival for un-backlogged CR. The number of CR attempting channel access is therefore $x+y$ with probability $Q_{a}(x, i) Q_{r}(y, i)$.

With a smaller group on each channel for channel access, contention among CR may further be alleviated by separation of CR traffic. We define the successful transmission function $\Omega(w, a, k)$ where its value represents the probability that there are $w$ successful transmission CRs out of $a$ concurrent attempting CRs over channel $1,2, \ldots, k$. With the channel selection algorithm $\Gamma=\left\{p_{k}\right\}$, we can find the probability that $w$ out of $a$ successfully wins channel contention as:

$$
\Omega(w, a, k)=\sum_{n=0}^{a} \Omega(w, a-n, k-1) p_{k}^{n}\left(1-g_{k}(n)\right)+\Omega(w-1, a-n, k-1) p_{k}^{n}\left(g_{k}(n)\right)
$$

$\Omega(w, a, k)$ can be recursively solved: Note that $\Omega(1, n, 0)=g_{0}(n)$ and $\Omega(0, n, 0)=1-$ $g_{0}(n)$. The contention avoidance/resolution function $g_{k}($.$) over channel k$ depends on the spectrum sensing mechanism (represented as channel availability $\phi_{k}$ ) and CSMA contention mechanism. Let $N_{c w}$ denote the contention window size, then $g_{k}($.$) can be therefore derived$ as:

$$
g_{k}(n)=\phi_{k} \sum_{i=0}^{N_{c w}-1}\left(\begin{array}{l}
n \\
1
\end{array}\right)\left(\frac{1}{N_{c w}}\right)\left(1-\frac{i+1}{N_{c w}}\right)^{n-1}
$$

Given $x$ new arrival, $y$ retransmission attempt, the probability of $w$ successful transmission can be represented as $\Omega(w, x+y, K) Q_{r}(y, i) Q_{a}(x, i)$. The number of remaining backlogged $C R$ in current frame is $i-y$, the number of backlogged CR after transmission attempt is $x+y-w$. For the resulting state $j$, the relation $j=x-w+y$ holds. Therefore, the transition probability of $\left\{X_{t}\right\}$ can be derived as:

$$
P_{i j}=\sum_{\substack{x+w+i=j \\ 0 \leq x \leq N-i, 0 \leq y \leq i, w \leq x+y}} \Omega(w, x+y, K) Q_{r}(y, i) Q_{a}(x, i)
$$

With the transition probability matrix $\mathbf{P}=\left\{P_{i j}\right\}$, we can solve the limiting probability $\boldsymbol{\Pi}=$ $\left\{\pi_{1}, \pi_{2}, \ldots, \pi_{\max (\mathrm{S})}\right\}$ of the Markov chain by $\boldsymbol{\Pi}=\boldsymbol{\Pi} \mathbf{P}$ and $\sum_{i} \pi_{i}=1$. The throughput can further be derived with the limiting probability $\Pi$, First we define the protocol efficiency $\eta$ as the portion that the MAC protocol utilizes channel capacity in a frame. Note that the frame duration is $t_{l}$; the duration for spectrum sensing is $t_{s}$; further access control (CSMA) over the channel requires a duration $t_{c}$. Therefore, $0 \leq \eta \leq \frac{t_{l}-t_{s}-t_{c}}{t_{l}}$. Let $b$ denote the number of backlogged CR and $\pi_{b}$ as its related limiting probability. The system throughput, defined as the aggregated bit transmitted over multichannel per frame can further been derived as in Eq.(9) and in Eq.(10), respectively.

\subsection{Steady state approximation}

Markov chain model suffers from the fact it provides no clear mathematical or physical meaning. Without losing intuition of protocol design and the hope of finding dependency among parameters for further performance enhancement, we apply a steady-state expectation approach to provide design insight. To consider the potential throughput of multichannel MAC, we analyse multichannel MAC under saturated load, controlled by Pseudo-Bayesian algorithm (Bertsekas \& Gallager, 1992) with parameter $p$. 


$$
\begin{aligned}
& R=\sum_{b=0}^{N} \sum_{j, x, y ; 0 \leq x \leq N-i, 0 \leq y \leq i, j \leq x+y} C \eta j \Omega(j, x+y, K) Q_{a}(x, b) Q_{r}(y, b) \pi_{b} \\
& U=\frac{1}{K} \sum_{b=0}^{N} \sum_{j, x, y ; 0 \leq x \leq N-i, 0 \leq y \leq i, j \leq x+y} j \Omega(j, x+y, K) Q_{a}(x, b) Q_{r}(y, b) \pi_{b}
\end{aligned}
$$

Define $X_{i}$ as the indicator of transmission attempt of $\mathrm{CR}_{i} ; K, A, B$ as the random variables denoting the selected channel, simultaneous transmitters (including $\mathrm{CR}_{i}$ ), and other co-channel $(K=k)$ transmitters in the same operating frame, respectively. For $\mathrm{CR}_{i}$ to successfully transmit its packet, the following conditions must hold: 1) $\mathrm{CR}_{i}$ decides to transmit. $\left(X_{i}=1\right.$.) 2) The selected channel $(K=k)$ must be free from PS appearance. 3$)$ $\mathrm{CR}_{i}$ wins CSMA contention $(S=1)$ with other $A=a$ out of $B=b$ co-channel transmitters. Let $P_{\text {succ }}^{(i)}$ denotes the probability of successful transmission for $\mathrm{CR}_{i} . P_{\text {succ }}^{(i)}$ is thus conditioned on $\left\{S, B, A, K, X_{i}\right\}$ :

$$
\begin{aligned}
P_{\text {succ }}^{(i)}= & \sum_{a, b, k} P\left[S=1, B=b, A=a, K=k, X_{i}=1\right] \\
= & \sum_{a, b, k} \cdot P\left[S=1 \mid B=b, A=a, K=k, X_{i}=1\right] \\
& \cdot P\left[B=b \mid A=a, K=k, X_{i}=1\right] \\
& \cdot P\left[A=a \mid K=k, X_{i}=1\right] \\
& \cdot P\left[K=k \mid X_{i}=1\right] \\
& \cdot P\left[X_{i}=1\right]
\end{aligned}
$$

At saturated load, CR is always queued with packet. Therefore, the probability that $\mathrm{CR}_{i}$ attempts to transmit is $p$. It is clear that, $P\left[X_{i}=1\right]=p$. The probability that $\mathrm{CR}_{i}$ selects channel $k$ is $P\left[K=k \mid X_{i}=1\right]=p_{k}$.

All CRs independently make access decision. The probability that there are $a$ simultaneous transmission attempts (including $\mathrm{CR}_{i}$ ) in the same operating frame is

$$
P\left[A=a \mid K=k, X_{i}=1\right]=\left(\begin{array}{c}
N-1 \\
a-1
\end{array}\right) p^{a-1}(1-p)^{N-a} .
$$

Given $\mathrm{CR}_{i}$ is at channel $k$ attempting transmission. The probability that there are exactly $b$ co-channel CR transmission attempts out of $a-1$ (excluded $\mathrm{CR}_{i}$ ) simultaneous transmission attempts in the same operating frame is

$$
P\left[B=b \mid A=a, K=k, X_{i}=1\right]=\left(\begin{array}{c}
a-1 \\
b
\end{array}\right)\left(\frac{1}{M}\right)^{b}\left(1-\frac{1}{M}\right)^{a-b-1} .
$$

For $\mathrm{CR}_{i}$ to "win" the channel in CSMA contention, the channel it selected must be free from PS occupation and the backoff time $C_{i}$ picks must be the smallest one of other $b \mathrm{CR}$. Thee 
contention window size is denoted by $N_{c w}$ and we can derive:

$$
\begin{aligned}
P[S=1 \mid B=b, & \left.A=a, K=k, X_{i}=1\right]= \\
& \left(1-q_{k}\right) \sum_{n=0}^{N_{c w}-1}\left(\frac{1}{N_{c w}}\right)\left(1-\frac{n+1}{N_{c w}}\right)^{b} .
\end{aligned}
$$

Then the normalized throughput $U$ / multichannel frame utilization is:

$$
\begin{aligned}
U & =\sum_{i} P_{\text {succ }}^{(i)} \\
& =N \sum_{a, b, k} P[S=1, B=b, A=a, K=k, X=1] .
\end{aligned}
$$

The second equality holds since all CRs follows a symmetric behaviour and we can remove the subscript $i$. It should be noted that the multichannel frame utilization is comparable to the normalized throughput in convention single channel MAC which is defined as average packet successfully transmitted frame/slot (per channel). The aggregated throughput $R$ is:

$$
R=N \sum_{a, b, k} \eta_{k} C_{k} P\left[S=1, B=b, A=a, K=k, X_{i}=1\right] .
$$

\section{Cognitive functionality and MAC protocol optimization}

CR brings new cognitive functionality for agile DSA. In CR multichannel MAC, the sensing capability of $\mathrm{CR}$ for retrieving environmental information and adaptation function among CRs open new degree of freedom in MAC protocol optimization. In this section, we return to the fundamentals of multichannel MAC problem under CR paradigm: 1) Collision avoidance/resolution 2) Channel Selection and develop related cognitive functionality of CR for MAC protocol optimization.

\subsection{Optimal collision avoidance/resolution for CR}

CSMA-based protocol suffers from its unstable nature, a stabilization mechanism is required and will significantly contribute to performance improvement. When there are too many CRs, every CR shall decrease its potential channel access in prevention of collision with other CRs, while CR shall increase the probability of channel access when there are much resource left.

From previous sections, $N$ and $\mathbf{q}$ can be acquired from the sensing function of $C R$ in the long run; $M, N_{c w}, \mathrm{C}$ and $\boldsymbol{\eta}$ are predetermined during CR design. Therefore, the throughput optimization in the proposed multichannel MAC protocol can be done by finding the optimal probability of transmission attempt $p^{*}$ that maximizes the aggregated throughput. Rewrite Eq.(16),

$$
R=\sum_{a=1}^{N} G(a) p^{a}(1-p)^{(N-a)}
$$

Where:

$$
\begin{aligned}
G(a)= & \sum_{b=0}^{a} \sum_{k=1}^{M} \sum_{n=1}^{N_{c w}-1} \eta_{k} C_{k}\left(1-q_{k}\right)\left(\frac{1}{N_{c w}}\right)\left(\frac{N_{c w}-n+1}{N_{c w}}\right)^{b} \\
& \cdot\left(\begin{array}{c}
N-1 \\
a-1
\end{array}\right)\left(\begin{array}{c}
a-1 \\
b
\end{array}\right)\left(p_{k}\right)^{b+1}\left(1-p_{k}\right)^{a-b-1}
\end{aligned}
$$


It should be noted that $G(a)$ is a non-decreasing function of $a$. In comparison to conventional single channel stabilization (Limb, 1987), information over diverse multichannel for both inter-system (CR-PSs) and intra-system (within CRN) is required to achieve stabilization over multichannel. Information retrieval can be done with the sensing function. The optimization problem can thus be formulated as :

Maximize

$$
R=\sum_{a=1}^{N} G(a) p^{a}(1-p)^{(N-a)}
$$

Subject to

$$
0 \leq p \leq 1
$$

Since the optimization parameter $p \in[0,1]$, Eq.(17) can be easily transformed and solved by interior-point method (Boyd \& Vandenberghe, 2004) to find the optimal probability of transmission attempt $p^{*}$. Another instinct method is to construct a look-up table. This table can be pre-determined and embedded in the design of CR. By periodically adapting (adaptation function) the probability of transmission attempt $p$ to $p *$, the expected throughput of CRs is then optimized. The proposed multichannel protocol is therefore optimized by cognitive functionalities - sensing function (inter-system information $\left(q_{k}\right)$ and intra-system information $(N)$ retrieval) and adaptation function (adapt to fit the environment.)

\subsection{Optimal channel selection for CR}

For $N$ CRs distributedly follows a selection algorithm $\Gamma=\left\{p_{k}\right\}$ for selecting $K$ channels for channel access, the optimization problem follows the similar method as the stabilization mechanism. Therefore, the throughput optimization in the proposed multichannel MAC protocol can be can be done by finding the optimal $p_{k}^{*}$ that maximizes the expected throughput. Rewrite Eq.(9), we have:

$$
R=\sum_{k-1}^{K} \sum_{a=1}^{N} \sum_{b=0}^{a} G^{\prime}(a, b) F(k) p_{k}^{b+1}\left(1-p_{k}\right)^{a-b-1}
$$

Where:

$$
\begin{gathered}
F(k)=\eta_{k} C_{k} \phi_{k} \\
G^{\prime}(a, b)=p^{a}(1-p)^{(N-a)\left(\begin{array}{c}
N-1 \\
a-1
\end{array}\right)\left(\begin{array}{c}
a-1 \\
b
\end{array}\right) \sum_{n=1}^{N_{c w}-1}\left(\frac{1}{N_{c w}}\right)\left(\frac{N_{c w}-n+1}{N_{c w}}\right)^{b}}
\end{gathered}
$$

The optimization problem can thus be formulated as follows:

Maximize

$$
R=\sum_{k-1}^{K} \sum_{a=1}^{N} \sum_{b=0}^{a-1} G^{\prime}(a, b) F(k) p_{k}^{b+1}(1-p)^{a-b-1}
$$

Subject to

$$
\sum_{k} p_{k}=1 \text { and } 0 \leq p_{k} \leq 1
$$



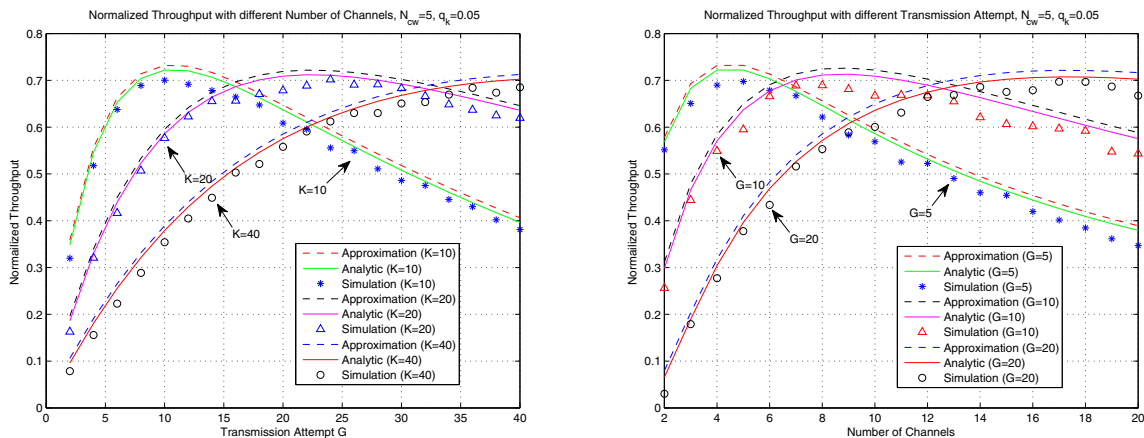

(a) Normalized Throughput $U$ with Different (b) Normalized Throughput $U$ with Transmission Number Channel $K$ via Transmission Attempt $G$, Attempt $G$ via Different Number of Channel $K$, $N_{c w}=5, q=0.05$ $N_{c w}=5, q=0.05$
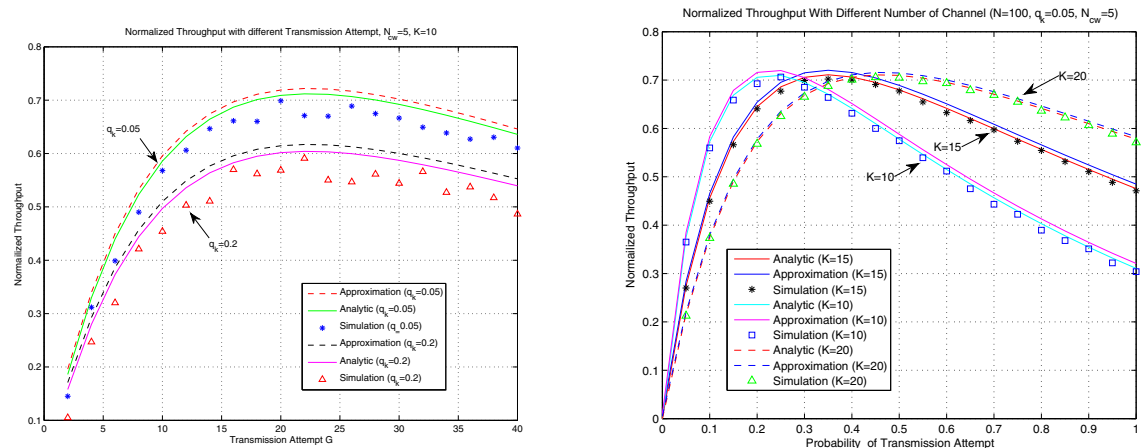

(c) Normalized Throughput $U$ with Different (d) Normalized Throughput $U$ with Different Probability of PS appearance $q_{k}, N_{c w}=5, K=10$ Number of Channel $K$ via Different Probability of Transmission Attempt $p, N_{c w}=5, K=10$

Fig. 8. Validation

It should be noted that Eq.(23)can be solved same as $p^{*}$ over convex domains. Eq.(23) provides a basic understanding about the optimal performance of channel selection algorithms. In the following section, we simulate different channel selection algorithms to provide some intuition of different selection algorithms.

\section{Simulation results}

\subsection{Validation}

First we validate and discuss the protocol performance. Fig. 8 illustrates the analytic and the simulation results of CR multichannel MAC. The simulation results (depicted in dots) validate our analytic model, providing a basic understanding about CR multichannel MAC performance. Note that in this part we turn off the cognitive functionality to analyse the original protocol performance. The results also suggest the correctness of the steady 
state approximation, which provides important insights for the protocol design and further optimization processes.

Fig. 8-(a) depicts the normalized throughput via CR attempt rate $G$. The simulation parameters are with $K=10,20,40 ; \Gamma=\left\{\frac{1}{R}\right\}, \eta=0.95, N_{c w}=5, q_{r}=0.2, q_{k}=0.05$. One can observe that with lower number of channel (resource), the MAC throughput will saturate faster (under lower attempt rate $G$ ), and then drop faster. The more the number of channel, the higher attempt rate the CR can support. It matches the intuition that with more resource, more CRs can be served. Without a proper stabilization mechanism, the normalized throughput under all $\lambda$ drops as attempt rate $G$ increased.

With different number of channels, different attempt rates of CR can be supported. Fig. 8-(b) illustrates the normalized MAC throughput via the number of channels. With a given transmission attempt $G$, when the number of channel is large, there are less contention when CRs selects over multichannel. On the other hand, the appearance of PSs' users will affect CR's multichannel MAC performance. In Fig. 8-(c), the higher the probability of PS appearance, the lower the CR multichannel MAC throughput will be achieved. Note that with the spectrum sensing, transmissions of PSs' users are protected.

The result in Fig. 8-(d) shows three cases when the number of $C R$ is much greater than the number of channels, that is, a crowded CRN; about the number of channels and less then the number of channels. It can be concluded that when the number of CR is less then the channel, it is reasonable to transmit it with higher probability when packet arrives. When the number of CR is higher, it should decrease the probability of transmission attempt $q$ to alleviate the contention over multichannel. The dramatic drop in throughput is more severe in a crowded CRN.

\subsection{Cognitive multichannel MAC performance}

For the proposed cognitive CSMA-based multichannel MAC protocol, the throughput outperforms the the conventional work of Aloha-based McMAC (Mo et al., 2008) with improvement in PS co-existence support and collision alleviation of CRs by CSMA. Fig. 9 shows the normalized/aggregated throughput via number of CRs and channels. (a) and (b) is the McMAC case while (c) and (d) is the cognitive multichannel MAC protocol. With the joint consideration of channel selection and contention avoidance/resolution, more sophisticated CR multichannel MAC and better normalized/aggregated throughput performance can be achieved. Note that the cognitive functionality is turned off for a fair comparison.

For normalized throughput/utilization of the cognitive multichannel MAC and McMAC respectively depicted in Fig. 9-(c) and Fig. 9-(a), co-channel intra-system contention in cognitive multichannel MAC is separated over multichannel and is further alleviated by the non-persistent CSMA, therefore, the throughput is enhanced. When the number of channel is low, this phenomena is significant. when the number of channel increase, the alleviation of co-channel contention is still valid in the improvement of channel utilization. The out performance comes from the smarter channel selection and the contention resolution among PS-CR and CR-CR.

The aggregated throughput performance is illustrated in Fig. 9-(d) and Fig. 9-(b). When the number of channel is increased, the throughput is first increased and then converged. It is because that $p$ and $N$ are fixed, CRs can not initiate more parallel transmissions even if there 
Average Utilization/ Normalized Throughput of M-MAC, $\mathrm{p}=0.4, \mathrm{q}=0.05, \mathrm{~N}_{\mathrm{cw}}=5$

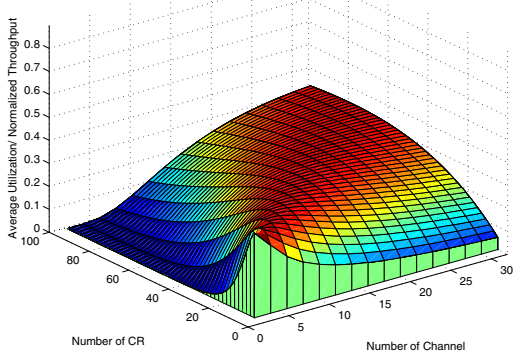

(a) Normalized Throughput/ Average Utilization of McMAC

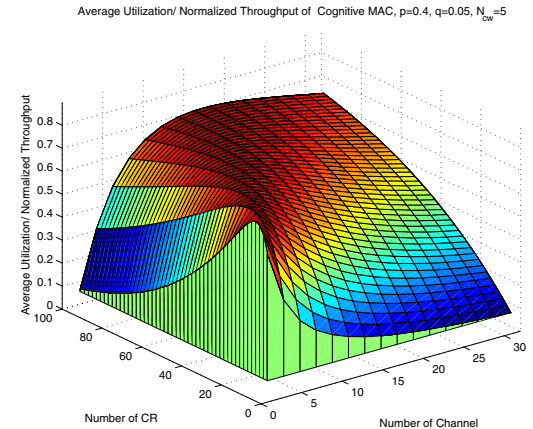

(c) Normalized Throughput/ Utilization of Cognitive MAC

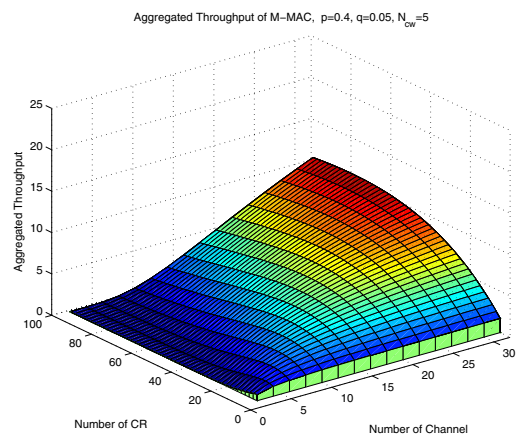

(b) Aggregated Throughput of McMAC

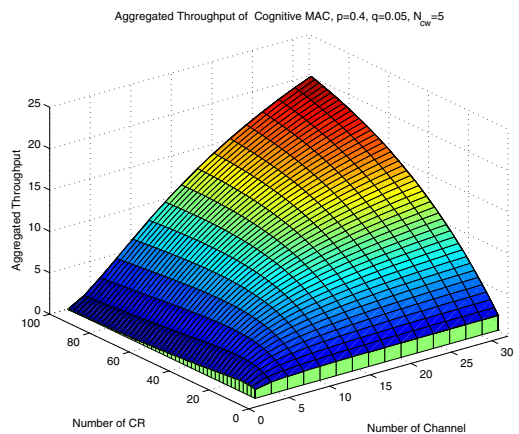

Average (d) Aggregated Throughput of Cognitive MAC

Fig. 9. Multichannel MAC performance

is still available resource. On the other hand, for a fix number of channels, increasing the size of CRN results in the increase of throughput since there are more CRs try to transmit, which is a feasible property of CR multichannel MAC.

\subsection{Optimal cognitive multichannel MAC}

With the cognizant information in Section 6, the proposed cognitive multichannel MAC can further been optimized. Fig. 10-(a) shows the normalized throughput with stabilization that maximizes throughput with $p *$. In addition to spectrum sensing and CSMA, with the help of cognizant information from PSs and CRN, the proposed cognitive multichannel protocol empowers CRN to adapt to the optimal probability of transmission attempt $p^{*}$ for pseudo-Bayesain algorithm. Without losing intuition, here we assume CRN without entering/leaveing CRs, i.e., no transient behaviour. In our simulation, we consider the case that the environmental information including the number of CR $N$, the probability of PS appearance $q_{k}$ is correctly and distributively acquired by all CRs in the long run. The channel capacity $C$ is set to unity and the size of contention window $N_{c w}=5$. The probability of PS 


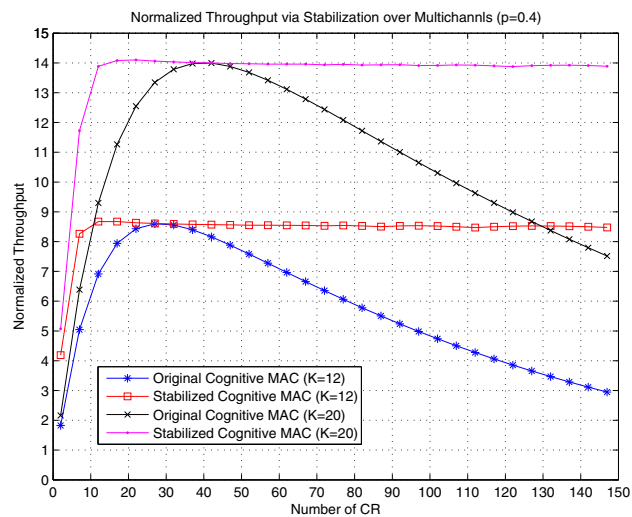

(a) Protocol Stabilization

Optimal Average Utilization/ Normalized Throughput of Cognitive MAC, $\mathrm{N}_{\mathrm{cw}}=5, \mathrm{q}_{\mathrm{k}}=0.05$

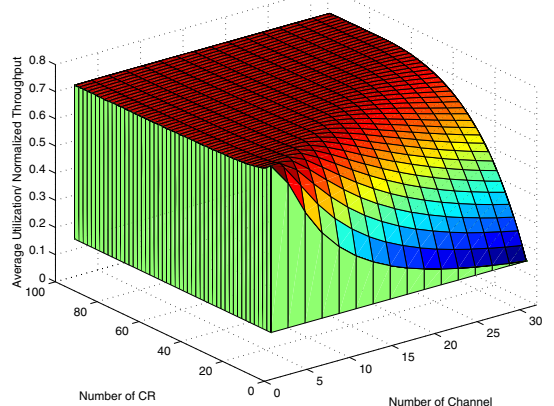

(c) Optimal Normalized Throughput/ Average (d) Optimized Utilization of Cognitive MAC

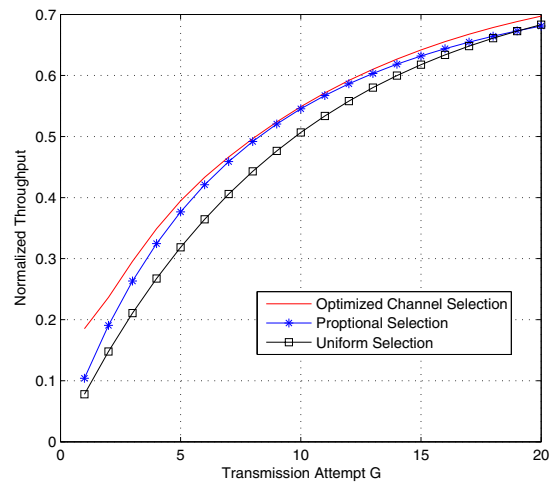

(b) Optimal Channel Selection

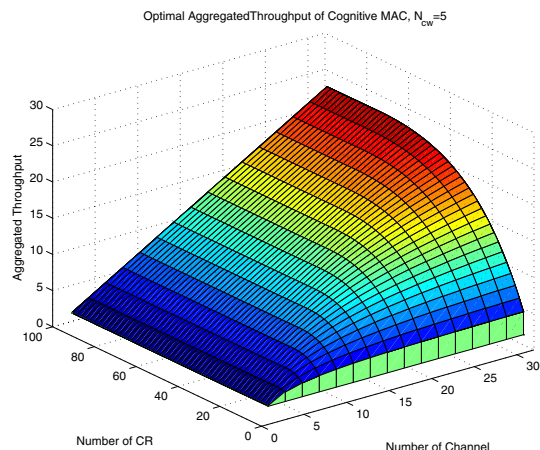

Aggregated Throughput of

Fig. 10. MAC Protocol Optimization with Cognitive Functionality

appearance is 0.05 . We simulate the case $K=12$ and $K=20$ as a representation of stabilization of the proposed cognitive multichannel MAC protocol.

Observing Fig. 10-(b), the optimal channel selection outperforms all other strategies. When the number of CR selections $N$ is low, its performance is equivalent to best selection, that is, the optimal selection algorithm selects the networks offering maximum utility. It should be noted that when $N$ increases, always selecting the best channel is no more the 'best' strategy. It is due to the fact that best selection saturates the best channels, leaving other channels far away from fully utilized.

Opportunistic access including random and proportional selection may be used to achieve expected utility improvement and load balancing. From the simulation results, proportional selection outperforms random selection the most of the time. When number of CR $N$ is not large, selecting best channels is a feasible manner, however, when $N$ is increasing, proportional channel selection is close to the optimal selection strategy. When the number of 


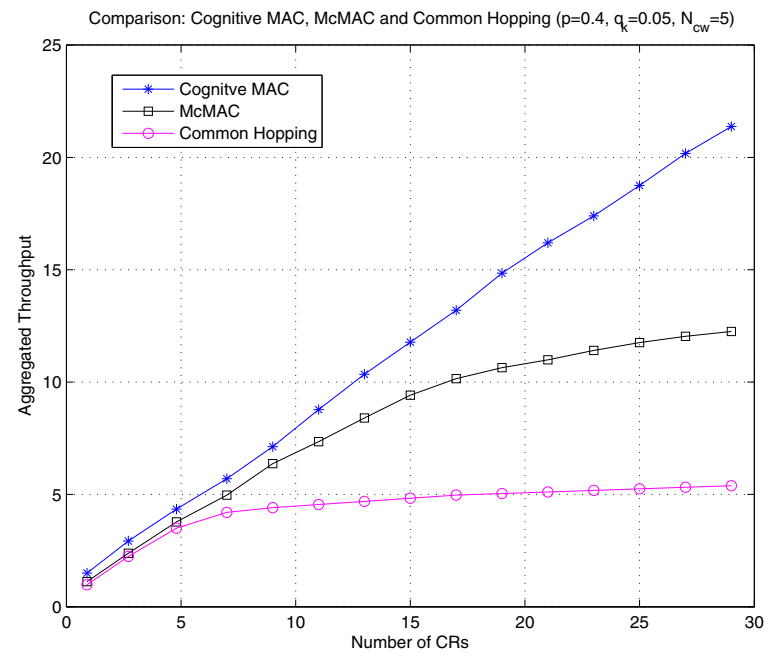

Fig. 11. Comparison of the proposed Cognitive MAC and other multichannel MAC

CR $N$ is high, random selection may be a good asymptotic channel selection strategy. While the number of $C R N$ is low, proportional selection will be a feasible approach.

Fig. 10-(c) and (d) depict normalized and aggregated throughput $R$ of the cognitive multichannel MAC with the cognitive functionalities for MAC protocol optimization. From the result, we further show that adaptation of $p^{*}$ and $\Gamma^{*}$ will optimize the throughput performance of multichannel MAC. The aggregated MAC throughput performance can be improved in comparison to the converged phenomena of non-cognitive protocol shown in Fig. 9. The average improvement is $27.4 \%$ under our simulation setting. The insight behind is : CR may increase its attempt probability and choose the better channel when system is in light load. While when the system is crowded, CR may adapt and selecting channel base on the optimization rule $p^{*}$ and $\Gamma^{*}$. MAC protocol optimization rely on the cognitive functionality, which will be a promising capability in future communication system such as CRN. From above, the system is stabilized by adapting $p^{*}$, therefore, the overall throughput is approaching the upper bound under all condition of CRN. Moreover, the upper bound is increased by optimal channel selection strategy $\Gamma^{*}$ of CR. This optimization further improves the original out-performance of the proposed cognitive multichannel MAC for CR.

In Fig. 11, the proposed cognitive multichannel MAC protocol is summarized out-performance over other protocol designs under all size of CR since it can increase $p$ to initiate more transmissions while there is more resource for the CRs and decrease $p$ to avoid contention and collisions while the resource is relatively rare. From above, the cognitive functionality and cognizant information of CR provide further possibility of protocol performance improvement. The speed and accuracy of information retrieval will affect the performance. More sophisticated information extracting/exchanging mechanisms left much room for future research. 


\section{Conclusion}

The future multichannel MAC under CR paradigm is very critical but challenging. CR must efficiently utilize the left spectrum resource while avoid interfering PSs' users. The key components in the multichannel MAC are collision avoidance/resolution and channel selection. In this chapter, a general multichannel MAC protocol scheme, the related mathematical model and the steady state approximation is proposed to build up the insight of CR multichannel MAC problem, solution and evaluation. The cognitive CSMA-based multichannel example suggests throughput enhancement for $\mathrm{CR}$ in comparison of current research status.

The cognitive functionality of cognitive radio makes future CR multichannel MAC more intelligent. We further develop and integrate the idea of cognition into MAC design of CRN. The proposed cognitive multichannel MAC protocol utilizes CRs' sensing and adaption functionality to extract both inter- and intra-system information and optimize the throughput performance. For both PSs' and CRs' operators, the proposed cognitive multichannel MAC protocol is feasible since it offers detection and avoidance for PSs, and provides diverse multichannel accessibility and adaptation that optimizes the MAC throughput performance. Simulation results further validate the cognitive functionality and show its significance. Future research in MAC for CR or cognition functionality are obviously crucial and interesting, and the cognitive multichannel MAC protocol for CRN is an exciting first step.

\section{References}

Akyildiz, I. F., Lee, W.-Y., Vuran, M. C. \& Mohanty, S. (2006). Next generation/dynamic spectrum access/cognitive radio wireless networks: a survey, Comput. Networks 50(13): 2127-2159.

URL: $h$ ttp://dx.doi.org/10.1016/j.comnet.2006.05.001

Al-Meshhadany, T. \& Ajib, W. (2007). New cdma-based mac protocol for ad hoc networks, Vehicular Technology Conference, 2007. VTC-2007 Fall. 2007 IEEE 66th pp. 91-95.

Bahl, P., Chandra, R. \& Dunagan, J. (2004). Ssch: slotted seeded channel hopping for capacity improvement in ieee 802.11 ad-hoc wireless networks, MobiCom '04: Proceedings of the 10th annual international conference on Mobile computing and networking, ACM, New York, NY, USA, pp. 216-230.

Bertsekas, D. \& Gallager, R. (1992). Data Networks, second edn, Prentice Hall.

Boyd, S. P. \& Vandenberghe, L. (2004). Convex Optimization, Cambridge University Press.

Chen, J., Sheu, S.-T. \& Yang, C.-A. (2003). A new multichannel access protocol for ieee 802.11 ad hoc wireless lans, Personal, Indoor and Mobile Radio Communications, 2003. PIMRC 2003. 14th IEEE Proceedings on 3: 2291-2296 vol.3.

Chen, K.-C., Peng, Y.-J., Prasad, N., Liang, Y.-C. \& Sun, S. (2008). Cognitive radio network architecture: part i - general structure, ICUIMC'08: Proceedings of the 2 nd international conference on Ubiquitous information management and communication, ACM, New York, NY, USA, pp. 114-119.

Cordeiro, C. \& Challapali, K. (2007). C-mac: A cognitive mac protocol for multi-channel wireless networks, New Frontiers in Dynamic Spectrum Access Networks, 2007. DySPAN 2007. 2nd IEEE International Symposium on pp. 147-157. 
Haykin, S. (2005). Cognitive radio: brain-empowered wireless communications, Selected Areas in Communications, IEEE Journal on 23(2): 201-220.

URL: $h$ ttp://dx.doi.org/10.1109/JSAC.2004.839380

IEEE (2009). IEEE Standard Committee 802.22 Working Group on Wireless Regional Area Networks, IEEE Std 802.22-2009 .

Jain, N., Das, S. \& Nasipuri, A. (2001). A multichannel csma mac protocol with receiver-based channel selection for multihop wireless networks, Computer Communications and Networks, 2001. Proceedings. Tenth International Conference on pp. 432-439.

Jakllari, G., Luo, W. \& Krishnamurthy, S. (2005). An integrated neighbor discovery and mac protocol for ad hoc networks using directional antennas, World of Wireless Mobile and Multimedia Networks, 2005. WoWMoM 2005. Sixth IEEE International Symposium on a pp. 11-21.

Jha, S., Rashid, M., Bhargava, V. \& Despins, C. (2011). Medium access control in distributed cognitive radio networks, Wireless Communications, IEEE 18(4): 41 -51.

Limb, K. K. J. O. (1987). Advances in Local Area Networks. Frontiers in Communications, IEEE Press.

Liu, X. \& Ding, Z. (2007). Escape: A channel evacuation protocol for spectrum-agile networks, New Frontiers in Dynamic Spectrum Access Networks, 2007. DySPAN 2007. 2nd IEEE International Symposium on pp. 292-302.

Marsan, M. \& Roffinella, D. (1983). Multichannel local area network protocols, Selected Areas in Communications, IEEE Journal on 1(5): 885-897.

Mitola, J., I. \& Maguire, G.Q., J. (1999). Cognitive radio: making software radios more personal, Personal Communications, IEEE 6(4): 13-18.

Mo, J., So, H.-S. \& Walrand, J. (2008). Comparison of multichannel mac protocols, Mobile Computing, IEEE Transactions on 7(1): 50-65.

Nasipuri, A., Zhuang, J. \& Das, S. (1999). A multichannel csma mac protocol for multihop wireless networks, Wireless Communications and Networking Conference, 1999. WCNC. 1999 IEEE pp. 1402-1406 vol.3.

NTIA (2003). U.s. frequency allocation.

URL: http://www.nitia.doc.gov/osmhome/allochrt.pdf

Report, F. \& Order (2002). Federal communication commission std., FCC 02: 48.

RoyChoudhury, R., Bandyopadhyay, S. \& Paul, K. (2000). A distributed mechanism for topology discovery in ad hoc wireless networks using mobile agents, MobiHoc '00: Proceedings of the 1st ACM international symposium on Mobile ad hoc networking $\mathcal{E}$ computing, IEEE Press, Piscataway, NJ, USA, pp. 145-146.

So, J. \& Vaidya, N. H. (2004). Multi-channel mac for ad hoc networks: handling multi-channel hidden terminals using a single transceiver, MobiHoc '04: Proceedings of the 5th ACM international symposium on Mobile ad hoc networking and computing, ACM, New York, NY, USA, pp. 222-233.

Sun, Y.-K., Chen, K.-C. \& Twu, D.-C. (1997). Generalized tree multiple access protocols in packet switching networks, Personal, Indoor and Mobile Radio Communications, 1997. 'Waves of the Year 2000'. PIMRC '97., The 8th IEEE International Symposium on 3: 918-922 vol.3.

Tang, Z. \& Garcia-Luna-Aceves, J. (1998). Hop reservation multiple access (hrma) for multichannel packet radio networks, Computer Communications and Networks, 1998. Proceedings. 7th International Conference on pp. 388-395. 
Timmers, M., Dejonghe, A., van der Perre, L. \& Catthoor, F. (2007). A distributed multichannel mac protocol for cognitive radio networks with primary user recognition, Cognitive Radio Oriented Wireless Networks and Communications, 2007. CrownCom 2007. 2nd International Conference on pp. 216-223.

Tzamaloukas, A. \& Garcia-Luna-Aceves, J. (2000). Channel-hopping multiple access, Communications, 2000. ICC 2000. 2000 IEEE International Conference on 1: 415-419 vol.1.

WC Hung, KLE Law, A. L.-G. (2002). A dynamic multi-channel mac for ad hoc lan, 21st Biennial Symposium on Communications .

Wu, S.-L., Lin, C.-Y., Tseng, Y.-C. \& Sheu, J.-L. (2000). A new multi-channel mac protocol with on-demand channel assignment for multi-hop mobile ad hoc networks, Parallel Architectures, Algorithms and Networks, 2000. I-SPAN 2000. Proceedings. International Symposium on pp. 232-237.

Zhang, L., Soong, B.-H. \& Xiao, W. (2003). A new multichannel mac protocol for ad hoc networks based on two-phase coding with power control (tpcpc), Information, Communications and Signal Processing, 2003 and the Fourth Pacific Rim Conference on Multimedia. Proceedings of the 2003 Joint Conference of the Fourth International Conference on 2: 1091-1095 vol.2.

Zhang, X. \& Su, H. (2011). Cream-mac: Cognitive radio-enabled multi-channel mac protocol over dynamic spectrum access networks, Selected Topics in Signal Processing, IEEE Journal of 5(1): $110-123$. 


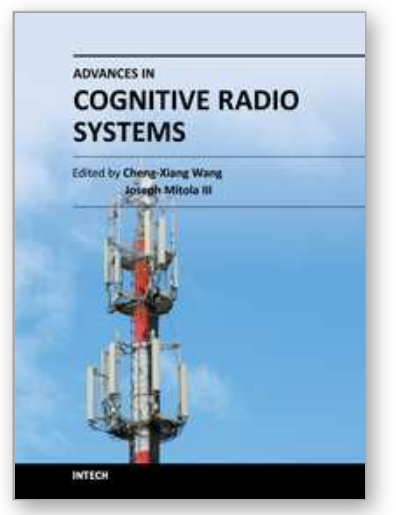

\section{Advances in Cognitive Radio Systems \\ Edited by Dr. Cheng-Xiang Wang}

ISBN 978-953-51-0666-1

Hard cover, 150 pages

Publisher InTech

Published online 05, July, 2012

Published in print edition July, 2012

\section{How to reference}

In order to correctly reference this scholarly work, feel free to copy and paste the following:

Po-Yao Huang (2012). Cognitive Media Access Control, Advances in Cognitive Radio Systems, Dr. ChengXiang Wang (Ed.), ISBN: 978-953-51-0666-1, InTech, Available from:

http://www.intechopen.com/books/advances-in-cognitive-radio-systems/cognitive-media-access-control

\section{INTECH}

open science | open minds

\section{InTech Europe}

University Campus STeP Ri

Slavka Krautzeka 83/A

51000 Rijeka, Croatia

Phone: +385 (51) 770447

Fax: +385 (51) 686166

www.intechopen.com

\section{InTech China}

Unit 405, Office Block, Hotel Equatorial Shanghai

No.65, Yan An Road (West), Shanghai, 200040, China

中国上海市延安西路65号上海国际贵都大饭店办公楼 405 单元

Phone: +86-21-62489820

Fax: $+86-21-62489821$ 
(C) 2012 The Author(s). Licensee IntechOpen. This is an open access article distributed under the terms of the Creative Commons Attribution 3.0 License, which permits unrestricted use, distribution, and reproduction in any medium, provided the original work is properly cited. 